\title{
Learning in honeybees as a function of amount and frequency of reward
}

\author{
GREGORY MCCLELLAN BUCHANAN and M. E. BITTERMAN \\ University of Hawaii, Honolulu, Hawaii
}

\begin{abstract}
In a series of four experiments with free-flying honeybees, individual foragers were trained with targets of two different colors that contained 5 or $20 \mu$ l of $50 \%$ sucrose solution. The two targets were singly presented in quasi-random sequences on each visit, with the amount of reward to be found on each target perfectly predictable from its color. The number of training visits (4-32) was varied both within and between experiments, and 80 also was the relative frequency of trials with the 5- and 20- $\mu$ l targets $(1: 1,2: 1,3: 1$, and 9:1). At the conclusion of training under each condition, unrewarded responses to the targets were measured in a 10-min extinction test, with the targets presented either separately to two different groups of animals (Experiment 1) or as a pair (Experiments 2-4). When the number of training trials with each target was the same (Experiments 1 and 2), the animals responded more in extinction to the 20- $\mu$ l target than to the $5-\mu \mathrm{l}$ target, although there was a decline in the overall level of responding to both targets (an overlearning-extinction effect) as the number of training trials increased. After nine times as many, or only three times as many, training trials with the 5- $\mu$ l target as with the $20-\mu l$ target, the animals responded more in extinction to the 5- $\mu$ l target (Experiment 3 ); after twice as many training trials with the $5-\mu l$ target as with the $20-\mu l$ target, there was equal responding to both (Experiment 4). The preferences shown in the choice tests of Experiments 2-4 could be simulated rather accurately on the assumptions of a model previously developed to deal with the discrete-trials choice behavior of honeybees and the further assumption that associative strength grows at a rate increasing with amount of reward to an asymptote independent of amount of reward.
\end{abstract}

In a continuing series of experiments on learning in honeybees as compared with learning in vertebrates (Bitterman, 1988), we turn now to the role of amount of reward, a powerful determinant of performance in appetitive conditioning experiments with vertebrates (Mackintosh, 1974). About quality of reward (concentration of sucrose solution in unlimited amount), we already have some interesting information from work with free-flying honeybees (Couvillon \& Bitterman, 1984; Shinoda \& Bitterman, 1987): (1) After training with $50 \%$ sucrose on one target and $20 \%$ sucrose on another, the $50 \%$ target is preferred in a choice test; (2) resistance to extinction increases at first and then decreases as a function of the number of training visits to $50 \%$ sucrose (the overlearning-extinction effect), but there is no hint of nonmonotonicity when the training concentation is $20 \%$; and (3) $20 \%$ sucrose is accepted less readily on a target that has always before contained $50 \%$ sucrose than on a target that has always before contained $20 \%$ sucrose (successive negative incentive contrast). These results are perfectly familiar, of course, from the study of vertebrate learning, although the underlying mechanisms may well be different. Successive negative incentive contrast, discovered early in mammals, has failed to appear in older

This research was supported by Grant BNS-8709785 from the National Science Foundation. Requests for reprints should be addressed to M. E. Bitterman, Békésy Laboratory of Neurobiology, 1993 EastWest Road, Honolulu, HI 96822. vertebrate lines, clearly suggesting convergence (Bitterman, 1975, 1988; Flaherty, 1982; Papini, Mustaca, \& Bitterman, 1988), which, as Simpson (1964) noted, is unlikely to be carried to the point of identity in "elaborately polygenic"' behavioral systems. A more direct indication of a difference in mechanism-and one that may be much more fundamental, because the results reported for honeybees are in conflict with those for all vertebrate species thus far studied-comes from experiments by Menzel and Erber (1972), who claimed that appetitive conditioning in honeybees is entirely independent of amount of reward.

The method employed by Menzel and Erber (1972) was to reward a forager in the presence of one color, then to give it an unreinforced choice test with that color and a different color, then to reward it again in the presence of the first color, test it again, and so forth. In one experiment, with a Y-maze, there were six rewards in an alley of one color, each followed by a test consisting of a series of $\mathbf{2 0}$ unreinforced choices between two differently colored alleys. The four groups of subjects were fed $5,10,25$, or $50 \mu l$ of a $50 \%$ sucrose solution on each training trial. In a second experiment, with differently colored targets on which free-flying animals could land and feed, there were seven rewarded training trials with one of the colors, each followed by an unreinforced choice test, $4 \mathrm{~min}$ long, in which all approaches to within $2 \mathrm{~cm}$ of each target were counted. Three groups of subjects were used, with the duration of feeding on the training trials equated over groups by varying the influx velocity 
of the sucrose solution so as to provide $2.5,9$, or $25 \mu \mathrm{l}$ of $30 \%$ sucrose in a 30 -sec period. The performance of the four groups in the first experiment was not significantly related to amount of reward. In the second experiment, there was a small mean difference in favor of the animals trained with the lowest influx velocity that was judged to be statistically significant on the basis of an improper test.

The explanation proposed by Menzel and Erber (1972) is that each pairing of signal and food "transmits"irrespective of the duration of feeding-a single "impulse" to the short-term memory system of the honeybee, but only if the feeding is continuous. If the feeding is interrupted repeatedly, as it was found to be with the lowest influx velocity in the second experiment, there is, in effect, a series of pairings on each trial, which makes for better subsequent performance when "the act of sucking lasts long enough" (p. 41) after each resumption for a transfer from short-term to long-term memory. "Long enough," on the basis of earlier work by Menzel (1968), means about $5 \mathrm{sec}$. Menzel and Erber (1972) assert that it is the sucking behavior, not the ingested sucrose, which is necessary for the transfer: "It is only important that the sucking continues; its result is irrelevant" (p. 41). This assertion seems to be based on their failure in preliminary experiments (which are not described) to find any effect of sucrose concentration over the range of $15 \%-60 \%$.

In our view, a more plausible explanation of Menzel and Erber's (1972) results for amount of reward is that the method they employed was insufficiently sensitive. For one thing, the number of rewarded responses was small, and the ratio of the number of unrewarded responses in the repeated extinction tests to the number of rewarded responses was so large that differences due to amount of reward may have been masked. Furthermore, each animal experienced only one of the several different amounts of reward, a procedure that often has given negative results in choice experiments with vertebrates; a more sensitive procedure is to have the animal choose between stimuli that have been paired with different amounts of reward (Mackintosh, 1974). Finally, as Menzel and Erber themselves recognized, animals trained with the different amounts of reward were tested at different points in the foraging cycle (foragers fed large amounts went to the hive before returning to be tested, but foragers fed small amounts remained in the experimental situation) - a confounding that should, if possible, have been avoided. A clear indication that Menzel and Erber's negative results for amount of sucrose solution cannot be taken at face value is provided by their negative results for sucrose concentration, which we now know to be an important parameter of appetitive conditioning in honeybees (Couvillon \& Bitterman, 1984). In the experiments reported here, we show that amount of sucrose also is an important parameter of appetitive conditioning in honeybees, and begin to inquire into its role in the conditioning process.
The index of associative strength in our experiments was resistance to extinction, and it may be useful before proceeding further to explain why we chose that index. Two other possibilities might immediately suggest themselves to students of vertebrate learning. One is latency of response, which, for free-flying bees, is not very reliable. The other is choice between two simultaneously presented targets, which works well when there is a single self-correction trial with feeding to repletion as the rewaid on each visit (Couvillon \& Bitterman, 1985, 1986, 1987,1988 ), but which pilot experiments showed to be too erratic for our purposes; given a series of small-reward trials on each visit, our animals often seemed to respond impulsively, without the hesitant inspection of both alternatives that characteristically precedes choice when the reward is feeding to repletion. We were compelled, therefore, to fall back on resistance to extinction, a measure previously put to good use (Couvillon \& Bitterman, 1980, 1982), but which has two rather serious disadvantages. One is that multiple groups are required to define the course of acquisition; in Experiment 2, for example, extinction was measured in one group of animals after 8 trials, in a second group after 16 trials, and so forth. The procedure of Menzel (1968) and Menzel and Erber (1972), which is to alternate training trials and extinction tests for a single group of animals, is inadequate, because it confounds the effects of training and extinction. Another disadvantage of resistance to extinction as an index of associative strength is that the relation is complicated by a process reminiscent of frustration in rats (Amsel, 1958); as has already been noted, resistance to extinction declines in the course of prolonged training under conditions that might be expected to produce high associative strength (Couvillon \& Bitterman, 1984). We planned the present experiments in the realization that an understanding of frustration might be necessary for a proper interpretation of the results, which themselves might contribute to that understanding.

\section{EXPERIMENT 1}

In this experiment, individual honeybees were given 32 trials with a target that always contained a $20-\mu$ l droplet of sucrose solution and an equal number of trials with a target of a different color that always contained a 5- $\mu$ l droplet of the same solution. Trials of the two kinds were intermixed on each visit in quasi-random fashion to balance for position in the foraging cycle. Then there was a single unrewarded test, with the 20- $\mu$ l target for one group of animals and the 5- $\mu \mathrm{l}$ target for another group, in which resistance to extinction was measured. The test was given at the outset of the final visit, immediately upon return from the hive.

\section{Method}

Subjects. The subjects were 24 honeybees (Apis mellifera) from our own hives situated near the laboratory. All were experimentally naive. 
Procedure. Individual honeybees were pretrained to fly from hive to laboratory and drink from a large drop (about $100 \mu \mathrm{l}$ ) of $50 \%$ sucrose solution on a target that was set on the sill of an open laboratory window. The target was a plastic disk, $5 \mathrm{~cm}$ in diameter and $1 \mathrm{~mm}$ thick, that was (nominally) half blue and half orange $\left(180^{\circ}\right.$ of each color). An animal selected at random from a group of foragers at a feeding station equipped with a jar of $10 \%-15 \%$ sucrose solution was captured in a matchbox, carried to the sill, and placed on the target. There it was permitted to drink its fill (during which time it was marked with a spot of lacquer) and then fly back to the hive. Typically, the animal would return to the laboratory in a few minutes, continuing to shuttle back and forth between the hive and the sill as long as food was available there. If the marked bee did not return to the sill after its first placement, it was picked up at the feeding station (where it usually could be found) and placed again on the target. The pretraining ended with the animal's second return to the sill of its own accord.

Arriving from the hive on each of 16 training visits, the animal found a target like the pretraining target, but all blue or all orange, containing either a $5-\mu \mathrm{l}$ or a $20-\mu \mathrm{l}$ droplet of $50 \%$ sucrose solution. After taking the sucrose, the animal would fly up from the target, hovering over the sill while the target was quickly removed and replaced with another target, again either blue or orange and containing either 5 or $20 \mu \mathrm{l}$ of the sucrose solution. The second target was quickly replaced by a third, and so on, until the animal left of its own accord for the hive. For 12 of the 24 subjects, the blue targets always contained $20 \mu \mathrm{l}$ of sucrose and the orange targets $5 \mu \mathrm{l}$, with the opposite true for the remaining subjects. The two targets were presented in balanced quasi-random sequences over the 16 training visits, during which each animal was scheduled to have about 32 trials with each target (an average of 2 trials with each target on each visit, with the expected average intake of about $50 \mu \mathrm{l})$. The procedure was such, of course, that the number of training trials with each target for each animal could not be fixed exactly, since it depended on the behavior of the animal, but the approximation was very close: The mean number of trials was 31.0 for the $20-\mu \mathrm{l}$ target and 31.1 trials for the 5- $\mu \mathrm{l}$ target.

Arriving from the hive on the 17 th visit, the animal found a fresh blue or orange target containing a $10-\mu$ l droplet of tap water (unacceptable, and distinguishable from the sucrose solution only by taste). Upon encountering the water, the animal would leave the target, return, leave again, return again (often only briefly, with no attempt to drink), and so forth, the interval between successive contacts with the target increasing as the test continued. All actual contacts with the target, however brief, during a 10-min extinction period were recorded by the experimenter, who pressed a button that activated a counter programmed to print stored frequencies at 30 -sec intervals. For the 12 animals of Group L (large), the color of the target in extinction was the 20- $\mu$ l training color (blue for 6 of the animals and orange for the rest). For the 12 animals of Group S (small), the 5- $\mu$ l training color was used in extinction.

\section{Results}

In Figure 1, the course of extinction in the two groups is plotted in terms of mean cumulative frequency of response over successive 30-sec intervals. Contrary to the findings of Menzel and Erber (1972), the curves show a clear effect of amount of reward-greater resistance to extinction in Group L than in Group S. Analysis of variance (based on discrete, uncumulated responses) yielded a significant groups effect $[F(1,14)=6.94, p<.0001]$, a significant effect of 2.5 -min blocks $[F(3,42)=46.41$, $p<.0001]$, and a nonsignificant interaction of groups $\times$ blocks $[F(3,42)=1.71, p=.1792]$.

Although, as already noted, amount of reward has been found to be a powerful determinant of performance in con-

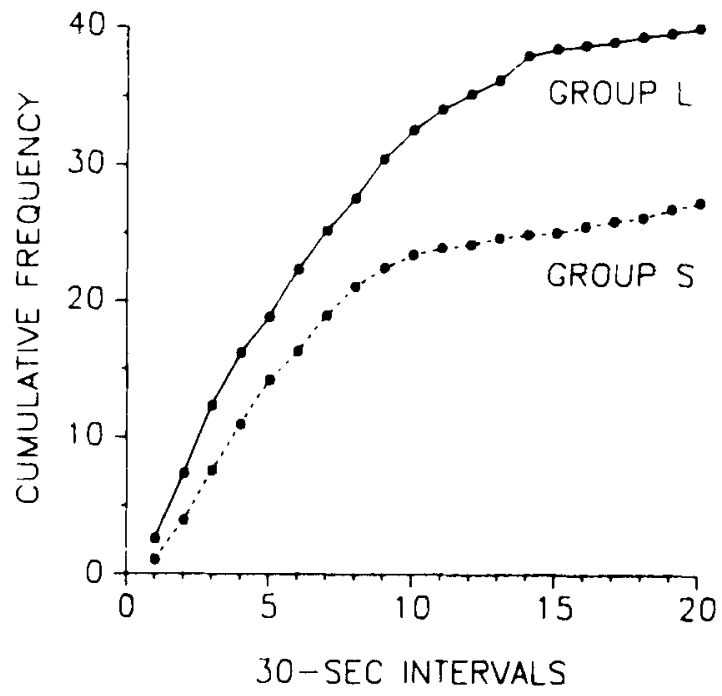

Figure 1. Mean cumulative responses in extinction to the 20- $\mu \mathrm{l}$ target (Group L) and the 5- $\mu$ l target (Group S) after approximately 32 training trials with each target (Experiment 1).

ditioning experiments with vertebrates, there has been litthe theoretical agreement as to how it works. Without pausing here to consider the merits of the various positions taken with respect to the vertebrate data, we simply draw on the vertebrate literature for some suggestions as to the role of amount of reward in the performance of honeybees. One possibility is that the animals learn about amount of reward as such-that training with different amounts of reward produces different memories or expectations (Tolman, 1932), which is to say, associations having different second terms or significates; orange may be associated with a large reward and blue with a small reward. For convenience, we describe this as the representational view, although it may be stated more objectively, as, for example, by Spence (1956), who conceived of the significates as anticipatory goal responses of different magnitude. Another (nonrepresentational) possibility is that the significates are the same-sucrose or sweetness in each case-but that the associations vary in strength as a function of amount, either asymptotically or in their rates of approach to a common asymptote, or even both (Rescorla \& Wagner, 1972). According to the representational theory, there was more response to the $20-\mu l$ color than to the 5- $\mu$ l color in the present experiment, not because of a difference in associative strength, but because of the greater valence of what Tolman called the "expectandum," or, in Hull-Spence terms, because the stronger anticipatory goal response interacted with habit strength to produce greater reaction potential. According to the more parsimonious nonrepresentational theory, the $20-\mu l$ training simply produced greater associative strength than the 5- $\mu$ l training.

\section{EXPERIMENT 2}

Again, in this experiment, honeybees were given equal numbers of training trials with differently colored $5-\mu l$ 
and $20-\mu l$ targets, but the procedure differed from that of Experiment 1 in two respects. First, the number of training trials was varied widely over groups. Disappearance of an initial preference for the $20-\mu \mathrm{l}$ color as the number of acquisition trials was increased would make it possible to reject both the representational theory and the unequal-asymptote version of the nonrepresentational theory. Second, a within-groups rather than a between-groups extinction test was used in the second experiment; after a given amount of training with each of the two targets separately, both targets were presented together without reinforcement, and the responses to each were counted. This change in procedure was made in the interest of efficiency: one group of animals, rather than two groups, was now required at each degree of training.

\section{Method}

Subjects. The subjects were 48 experimentally naive foragers from our own hives. They were assigned at random to four equal groups.

Procedure. The pretraining procedure was exactly the same as in Experiment 1, and so also was the training procedure, except that the number of training visits varied over groups -4 visits for Group 4, 8 visits for Group 8, 16 visits for Group 16, and 32 visits for Group 32. Again, it was expected that the animals would aver-

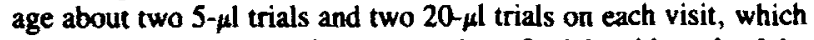
meant that Group 4 would average about 8 trials with each of the targets; Group 8, about 16 trials with each; Group 16, about 32 trials with each; and Group 32, about 64 trials with each. The mean numbers of 5- and $20-\mu \mathrm{l}$ trials actually given were 8.1 and 8.0 for Group 4; 16.6 and 16.6 for Group 8; 31.2 and 31.1 for Group 16; and 63.3 and 63.5 for Group 32. As before, the 5- $\mu$ l target was orange and the $20-\mu l$ target was blue for half the animals in each group; for the rest, the color-amount relation was reversed. After its last training visit, each animal returned from the hive to find two fresh targets, one blue and the other orange, set $10 \mathrm{~cm}$ apart in a lateral arrangement on the window sill. For 6 animals in each group, the 5- $\mu$ l target (blue for 3 and orange for 3 ) was at left, and

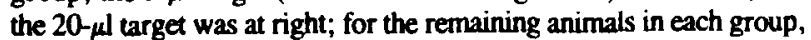

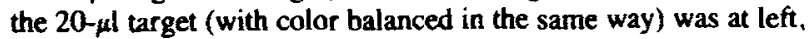
and the 5- $\mu$ l target was at right. Each target now contained a $10-\mu 1$ droplet of tap water, and responses to each were recorded in a 10min period.

\section{Results}

In Figure 2, the mean number of responses in extinction made by the animals of each group to each target is plotted as a function of the number of acquisition trials. The curves show a preference for the $20-\mu l$ target that persists as the number of acquisition trials increases, along with a decline in the overall level of responding to both targets. Analysis of variance yielded a significant effect of amount of reward $[F(1,40)=11.42, p=.0016]$, a significant effect of number of acquisition trials $[F(3,40)$ $=4.42, p=.0090]$, and a nonsignificant interaction of amount of reward $\times$ number of acquisition trials $(F<1)$.

If the preference for the $20-\mu l$ target had disappeared as the number of training trials increased, we should have been able to reject the representational theory as well as the unequal-asymptote version of the nonrepresentational theory, both of which predict that asymptotic attractive-

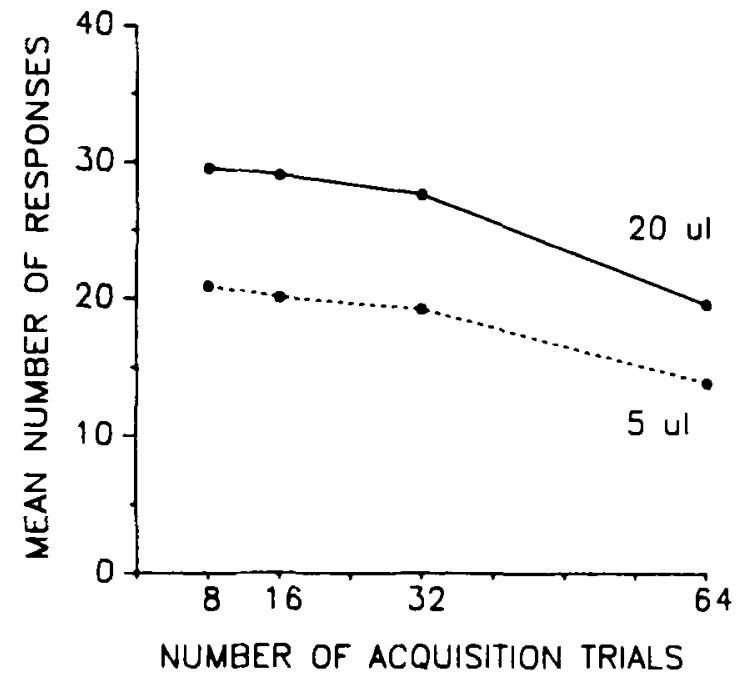

Figure 2. Mean number of responses in extinction to the simultaneously presented $20-\mu \mathrm{l}$ and $5-\mu \mathrm{l}$ tangets after approximately 8,16 , 32, or 64 training trials with each target (Experiment 2).

ness will increase with amount of reward. The persistence of the preference does not, however, make it possible to reject the equal-asymptote version of nonrepresentational theory, because the training may not have been carried far enough. If small rewards sustain only very low rates of growth in associative strength, considerably more than 32 training visits-perhaps even twice that number-might be required for definitive asymptotic information, which would be extremely costly in view of the many distracting and disruptive influences to which honeybees may be subjected in the course of 5 or $6 \mathrm{~h}$ of foraging. In Experiments 3 and 4 , we looked for a more cost-effective approach to the problem.

The progressive decline in overall level of responding in extinction as the number of training trials increased in the present experiment is reminiscent of the overleamingextinction effect previously found with feeding to repletion on $50 \%$ (but not $20 \%$ ) sucrose solution (Couvillon \& Bitterman, 1984; Shinoda \& Bitterman, 1987). Again, there are two ways to try to deal with such results on the basis of frustration theory (Amsel, 1958), one representational and the other nonrepresentational. According to the representational view, frustration is generated by a discrepancy between the remembered consequences of response (in our experiments, sucrose solution of a given volume and concentration) and the new consequences (water). According to the nonrepresentational view, frustration is generated by a discrepancy between the associative strength developed in training with the old consequences and the associative strength that can be supported by the new ones (Daly \& Daly, 1982). The present data do not seem to yield readily to either interpretation, pointing again to the need for a careful experimental analysis of the overlearning-extinction effect, after training both with single and with multiple stimuli, and in singlestimulus as well as in choice tests. 


\section{EXPERIMENT 3}

In the two previous experiments, the animals had equal numbers of training trials with the 5- $\mu \mathrm{l}$ and $20-\mu \mathrm{l}$ targets, but in the present experiment, amount and frequency of reward were opposed; that is, there were more trials (three or nine times as many) with the 5- $\mu$ l target than with the $20-\mu l$ target. The purpose was to provide further information about the way in which associative strength grows as a function of amount of reward, with special attention to the question of asymptotic equality.

\section{Method}

Subjects. The subjects were 36 experimentally naive foragers from our own hives. They were assigned at random to three equal groups.

Procedure. The pretraining procedure was the same as in the preceding experiments, and so also was the training, except for variations in the number of visits and the number of trials with each target. Group 16R9 had 16 training visits with a planned 9:1 ratio of small to large rewards; there were to be $820-\mu$ l rewards (one every second visit) on a target of one color (orange or blue) and $725-\mu l$ rewards (the actual mean number was 73.3 ) on a target of the alternative color. Group 16R3, too, had 16 training visits, but with a planned 3:1 ratio of small to large rewards; there were to be $1620-\mu 1$ rewards (one on each visit) and $485-\mu l$ rewards (the actual mean number was 47.6). Group 32R3 was trained as was Group 16R3, with a planned 3:1 ratio, but it had twice the number of visits; there were to be $3220-\mu l$ rewards (one on each visit) and $965-\mu l$ rewards (the actual mean number was 97.2). As in the preceding experiments, the larger reward was presented on a blue target for half the animals and on an orange target for the rest. The procedure in the 10 -min extinction test (given on each animal's return after the last training visit) was the same as in Experiment 2; fresh blue and orange targets, now baited with $10-\mu \mid$ droplets of tap water, were presented in lateral arrangements that were balanced over subjects.

\section{Results}

In Figure 3, the course of extinction in the three groups is plotted in terms of the mean cumulative number of responses to each target over successive 30-sec intervals. All groups responded less to the 20- $\mu$ l target than to the more frequently rewarded 5- $\mu$ larget, the difference being greatest in Group 16R9 and smallest in Group 32R3. Analysis of variance yielded a significant effect of amount of reward $[F(1,30)=20.26, p=.0001]$, a significant groups effect $[F(2,30)=14.82, p=.0356]$, and an interaction of groups $x$ amount of reward that is not quite significant at the conventional $5 \%$ level $[F(2,30)=3.02$, $p=.0639$ ].

These data, together with those of Experiment 2, can be explained without great difficulty in equal-asymptote, nonrepresentational terms-on the assumption, specifically, that associative strength approaches a common asymptote at twice the rate when the amount of reward is $20 \mu \mathrm{l}$ than when it is $5 \mu \mathrm{l}$. Before the details of the explanation are given, however, it is convenient to describe an experiment designed to test that assumption, which can be appreciated apart from those details.
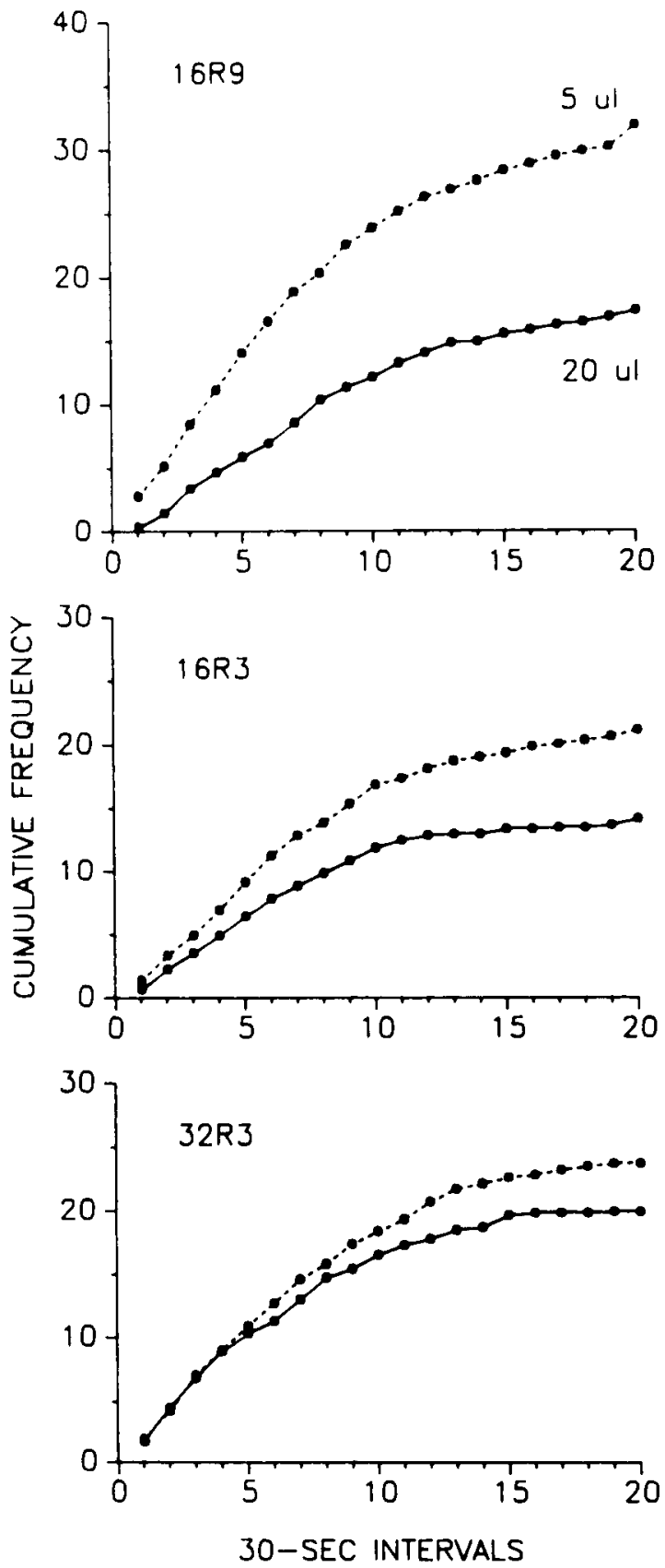

Figure 3. Mean number of responses in extinction to the simultaneously presented $20-\mu$ and 5- $\mu$ targets after training with both targets (Experiment 3). Group 16R9, approximately $820-\mu$ and 725 -

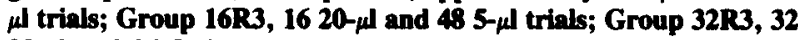
$20-\mu l$ and $965-\mu l$ trials.

\section{EXPERIMENT 4}

Again, in this experiment, amount and frequency of reward were opposed. In this case, however, there were only two trials with the 5- $\mu$ l target for each trial with the $20-\mu l$ target, a ratio predicted to produce equal associative strengths. 


\section{Method}

Subjects. The subjects were 16 experimentally naive foragers from our own hives. They were assigned at random to two equal groups.

Procedure. The pretaining and training procedures were the same as in the preceding experiments, except for the scheduling of training visits and trials. For the animals of Group 9R2, there were to be nine visits with a total of $1220-\mu$ l trials and $245-\mu$ l trials (the actual mean numbers were 12.0 and 24.9). For the animals of Group 19R2, there were to be 19 visits with a total of $2520-\mu$ l trials and $505-\mu$ l trials (the actual mean numbers were 24.7 and 50.9). The extinction procedure, which involved the presentation of fresh orange and blue targets in balanced lateral arrangements, was the same as in Experiments 2 and 3.

\section{Results}

In Figure 4, the course of extinction in the two groups is plotted in terms of the mean cumulative number of responses to each target over successive 30 -sec intervals. As predicted, the number of responses to the two targets was almost identical, both after the smaller number of training visits and after the larger number of training visits. Analysis of variance yielded a nonsignificant groups effect $[F(1,14)=2.06, p=.1727\}$, a nonsignificant effect of amount of reward $(F<1)$, and a nonsignificant interaction of groups $\times$ amount of reward $(F<1)$.

\section{GENERAL DISCUSSION}

In Experiment 1, amount of reward was found to play a substantial role in the appetitive conditioning of honey-

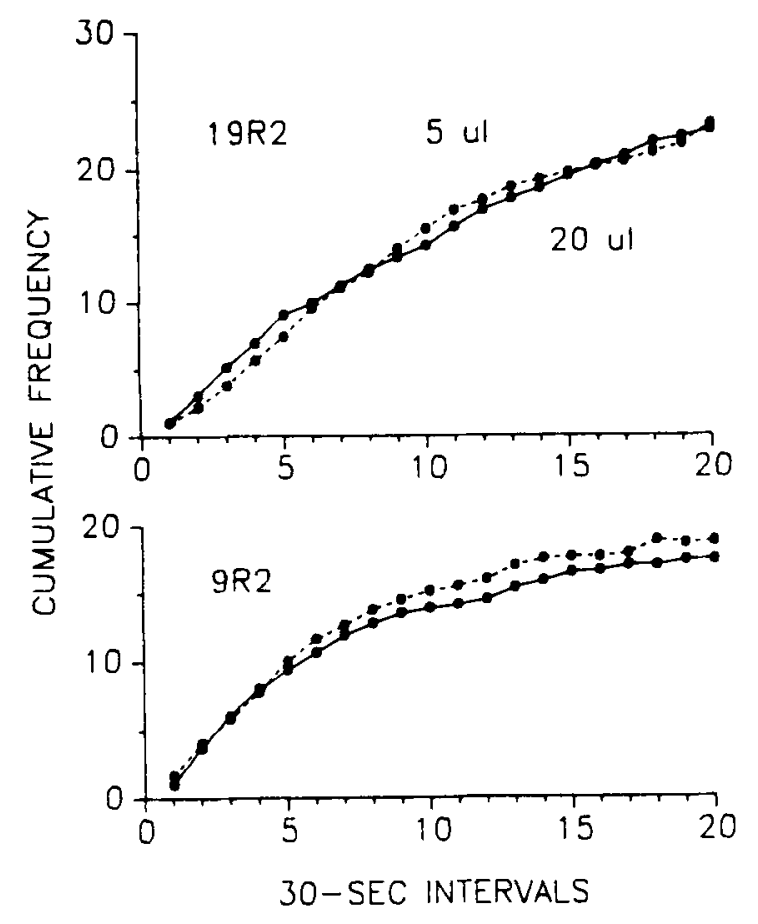

Figure 4. Mean number of responses in extinction to the simultaneously presented $20-\mu \mathrm{l}$ and $5-\mu$ largets after training with both targets (Experiment 4). Group 19R2, approximately 25 20- $\mu$ and 50

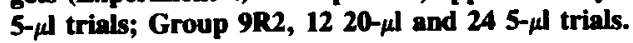

bees. In Experiments 2-4, which were designed to help us choose among various conceptions of that role, results were obtained that, as already noted, yield readily to an equal-asymptote, nonrepresentational analysis.

Plotted in Figure 5 are two curves showing how the associative strength of each target is assumed to grow as a function of the number of rewarded trials. The change is expressed by a linear equation (Bush \& Mosteller, 1951) in the familiar notation of Rescorla and Wagner (1972),

$$
\Delta V=\beta(\lambda-V) \text {, }
$$

with $V$ representing associative strength, $\Delta V$ the increment in associative strength produced by reward, $\lambda$ the asymptotic associative strength, and $\beta$ the learning-rate parameter. For purposes of simplicity in this most preliminary analysis, we use $V$ to represent the total associative strength of each target, without bothering to distinguish between unique properties and properties common to the two targets. The two curves are for $\beta=.02$ and $\beta=.04$, with $\lambda=1$ in each case. It will be noted that the curves originate at $V=.3$, which is assumed to be the associative strength produced by feeding to repletion in the pretraining; the value is provided by the work of Couvillon and Bitterman $(1985,1986,1987,1988)$, who have successfully modeled the performance of honeybees in a wide range of discrete-trials choice problems after just such pretraining. (The results of their experiments, in which correct choice always was rewarded by feeding to repletion on $50 \%$ sucrose, were fitted with $\lambda=1$ and incremental $\beta$ on the order of .2). Shown also in Figure 5 are the associative strengths of the two alternatives that would be generated in the training of each of the nine groups of Experiments 2-4 on the assumption that $\beta=.02$ for the 5- $\mu \mathrm{l}$ reward and $\beta=.04$ for the $20-\mu \mathrm{l}$ reward. From these associative strengths, the obtained proportions of response to the $20-\mu \mathrm{l}$ alternative in the extinction tests can be predicted in the following way:

We begin with Couvillon and Bitterman's assumption that the probability of choosing alternative $A$ rather than alternative $B$ is a function of its relative associative strength, $r_{A}=V_{A} /\left(V_{A}+V_{B}\right)$, where $V_{A}$ is the associative strength of alternative $A$ (here the 20- $\mu$ l target) and $V_{B}$ is the associative strength of alternative $B$ (here the 5- $\mu$ l target). The computed $R_{A}$ values for Groups $4,8,16$, and 32 of Experiment 2 at the end of training are $.55, .56$, .56 , and .54 , respectively; for Groups $16 \mathrm{R} 9,16 \mathrm{R} 3$, and 32R3 of Experiment 3, .37, .46, and .47, respectively; and for Groups 19R2 and 9R2 of Experiment 4, .50 and .49 , respectively. Next, we turn to one of a set of closely similar choice functions-functions relating $P_{A}$ (the probability of choosing alternative $A$ rather than alternative $B$ ) to $r_{A}$-that were used by Couvillon and Bitterman in the successful simulation of their discrete-trials data. Described in terms of its scaling parameters as $K=.75$, $s=.625$, the function is plotted in Figure 6 with only the values for $r \geq .5$ shown because it is perfectly symmetrical for $r<.5$. The shape of the function is sigmoidal and 


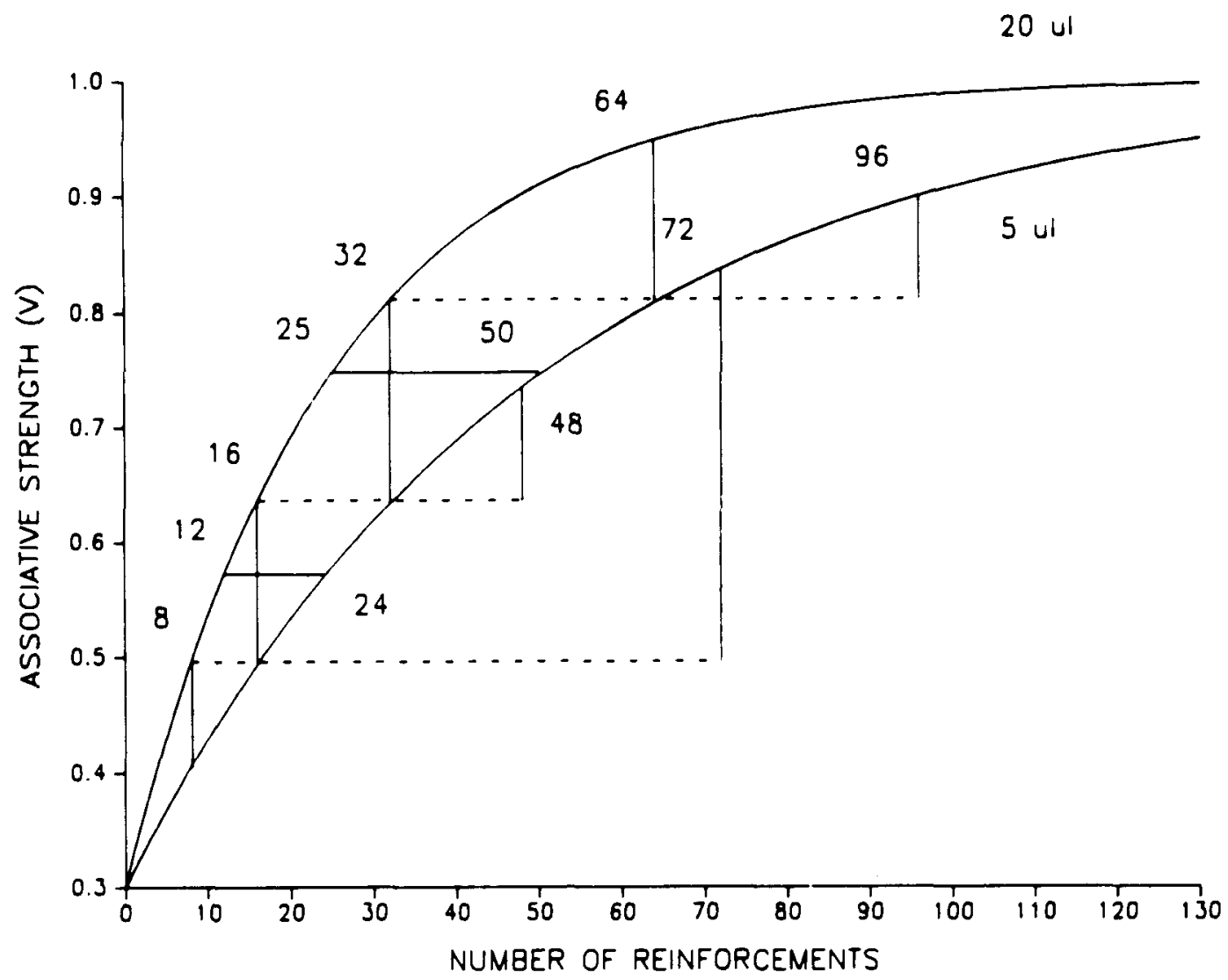

Figure 5. Hypothetical growth of anociative strength $\mathrm{s}$ a function of the number of rewarded trials to a common asymptote $(\lambda=1)$ at different rates $(\beta=.04$ for $20 \mu$ and .02 for $5 \mu)$. The nociative strengthe of the 5 - and $24-\mu$ sinernattves generated in the training of each of the nine groups of Experiments 2-4 abo are stown, the two values for each group being connected by straight lines.

its steepness is considerably greater than that of the linear ("matching") function $(K=1, s=.5)$ which is plotted along with it for purposes of comparison and which provides a substantially poorer fit to Couvillon and Bitterman's discrete-trials data. For each value of $r_{A}$, we read the corresponding probability of choosing the $20-\mu \mathrm{l}$ stimulus, $P_{A}$, from the choice-function, and from $P_{A}$ we can predict the test results if we are willing to assume that the determinants of choice at the outset of extinction also determine the distribution of responses to the two stimuli over the entire 10-min extinction period (during which the associative strengths of both stimuli are changing rapidly as a function of the choices actually made). Figure 7 shows that there is indeed a close correlation between the predicted proportions of response to the 20- $\mu \mathrm{l}$ target in the nine extinction tests of Experiments 2-4 and the proportions actually obtained. The Pearson coefficient is .97 .

Given this striking agreement of theory and data, it is perhaps necessary to emphasize that the two acquisition functions we used were not selected on the basis of an exhaustive parametric search. Other pairs of functions,

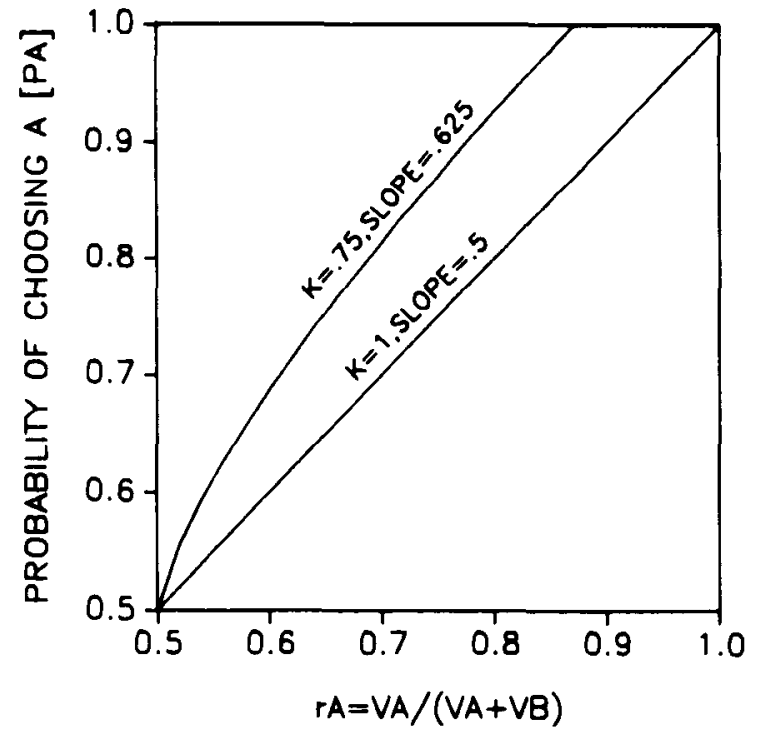

Figure 6. Two functions for predicting the probability of choosing allernattve $A\left(P_{A}\right)$ on the becis of the relative anociative treagth

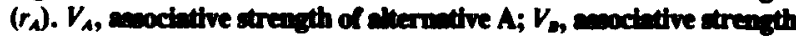
of alternative $\mathbf{B}$. 


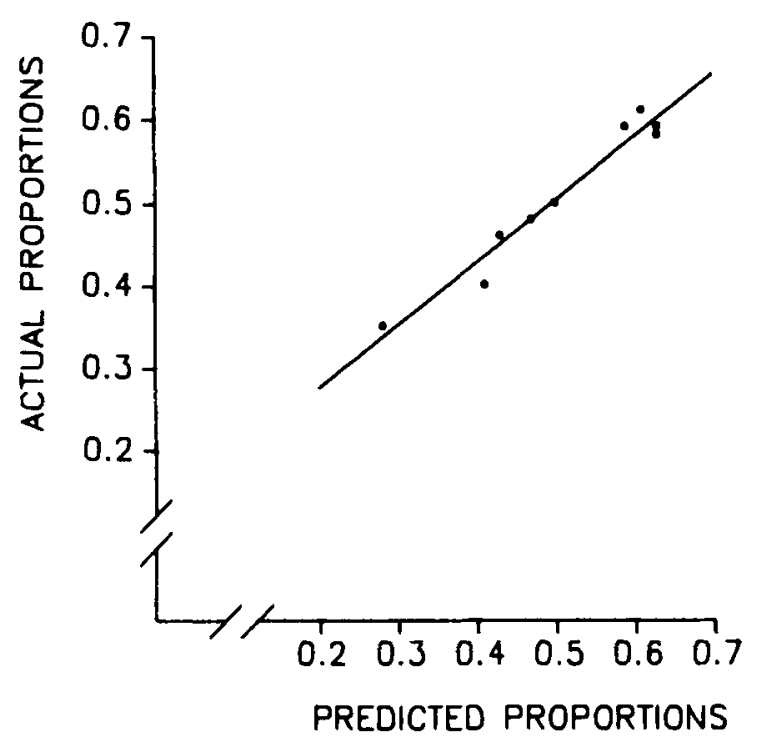

Figure 7. The relation between obtrined and predicted proportions of response to the 20- $\mu$ alternative by the nine groups of Experiments 2-4.

growing either to a common asymptote at different rates or to different asymptotes at a common rate, might fit our rather sparse data just as well, although we did not spend much time looking for them because they could hardly have been expected to yield an appreciably better fit. We are satisfied now to argue merely that equal-asymptote, nonrepresentational theory cannot be rejected, and that the best strategy would be to try in subsequent experiments to find ways of constraining it further, if only by brute increase in the number of training trials. What will be required in any case is a proper moment-by-moment theory of extinction that will predict not only choice ratios, but absolute levels of responding. Always in the background of our thinking has been the possibility that the decline in resistance to extinction with increasing frequency of reward that has appeared in some of our experiments can be understood in terms of a model like that developed by Daly and Daly (1982) to deal with an array of what seem to be frustration-based phenomena in rats, but the possibility may be more difficult to realize than we imagine. The Daly model itself has thus far been directed only at various qualitative features of the rat data and has yet to be tested quantitatively.

As to how the role of reward in the learning of honeybees may differ from its role in the learning of vertebrates, there is little to be said, not only because we know so little about learning in honeybees, but also because we know so little about learning in vertebrates. Although there have, of course, been a great many competent and interesting experiments with vertebrates (mostly rats) on acquisition and extinction as a function of almost every conceivable reward parameter (Mackintosh, 1974), the work has not been sufficiently systematic or sustained to pro- vide any basis for choice even among such relatively broad conceptions as we have considered here. In a time when the importance of a disciplined, quantitative approach to the problem was first coming to be appreciated (Hull, 1943, 1952), the necessary computational resources were not available; now that they are, critical interest in the problem has waned.

\section{REFERENCES}

AMSEL, A. (1958). The role of frustrative nonreward in noncontinuous reward situations. Psychological Bulletin, 55, $102-119$.

Bitterman, M. E. (1975). The comparative analysis of learning. Science, 188, 699-709.

BrTtERMAN, M. E. (1988). Vertebrate-invertebrate comparisons. In H. J. Jerison \& I. L. Jerison (Eds.), Intelligence and evolutionary biology (pp. 251-276). Berlin: Springer-Verlag.

Bush, R. R., MOSTELLER, F. (1951). A mathematical model for simple learning. Psychological Review, 58, 313-323.

Couvillon, P. A., a Bitterman, M. E. (1980). Some phenomena of associative leaming in honeyboes. Joumal of Comparative \& Physiological Psychology, 94, 878-885.

Couvillon, P. A., Bitterman, M. E. (1982). Compound conditioning in honeybees. Joumal of Comparative \& Physiological Psychology, 96, 192-199.

Couvillon, P. A., \& Bittrerman, M. E. (1984). The overlearningextinction effect and successive negative contrast in honeybees (Apis mellifera). Joumal of Comparative Psychology, 98, 100-109.

Couvillon, P. A., Bitterman, M. E. (1985). Analysis of choice in honeybees. Animal Learning \& Behavior, 13, 246-252.

Couvillon, P. A., \& Bitterman, M. E. (1986). Performance of honeybees in reversal and ambiguous-cue problems: Tests of a choice model. Animal Leaming \& Behavior, 14, 225-231.

Couvillon, P. A., Bitterman, M. E. (1987). Discrimination of color-odor compounds by honeybees: Tests of a continuity model. Animal Leaming \& Behavior, 15, 218-227.

Couvillon, P. A., \& Bitterman, M. E. (1988). Compound-component and conditional discrimination of colors and odors by honeybees: Further tests of a continuity model. Animal Learning \& Behavior, 16, 67-74.

DALY, H. B., DAlY, J. T. (1982). A mathematical model of reward and aversive nonreward: Its application in over 30 appetitive learning situations. Journal of Experimental Psychology: General, 111, 441-480.

FLAHERTY, C. F. (1982). Incentive contrast: A review of behavioral changes following shifts in reward. Animal Learning \& Behavior, 10 , 409-440.

Hull, C. L. (1943). Principles of behavior. New York: AppletonCentury-Crofts.

HuLl, C. L. (1952). A behavior system. New Haven, CT: Yale University Press.

MACKINTOSH, N. J. (1974). The psychology of animal learning. London: Academic Press.

MENZeL, R. (1968). Das Gedächtnis der Honigbiene für Specktralfarben: I. Kurzzeitiges und langzeitiges Behalten. Zeitschrift fur vergleichende Physiologie, 60, 82-102.

Menzel, R., \& ERBER, J. (1972). The influence of the quantity of reward on the learning performance in honeybees. Behavior, 41, 27-42.

Papini, M. R., Mustaca, A. E., Bitterman, M. E. (1988). Successive negative contrast in the consummatory responding of didelphid marsupials. Animal Learning \& Behavior, 16, 53-57.

Rescorla, R. A., Wagner, A. R. (1972). A theory of Pavlovian conditioning: Variations in the effectiveness of reinforcement and nonreinforcement. In A. H. Black \& W. F. Prokasy (Eds.), Classical conditioning II: Current research and theory (pp. 64-99). New York: Appleton-Century-Crofts. 
Shinoda, A., Brterman, M. E. (1987). Analysis of the overlearning extinction effect in honeybees. Animal Leaming \& Behavior. 15. 93-96

StMpson, C. G. (1964). Organisms and molecules in evolution. Science, 146, 1535-1538.

SPENCE, K. W. (1956). Behavior theory and conditioning. New Haven, CT: Yale University Press.
Tolman, E. C. (1932). Purposive behavior in animals and men. New York: Century.

(Manuscript received November 13, 1987; revision axcepted for publication March 1, 1988.) 